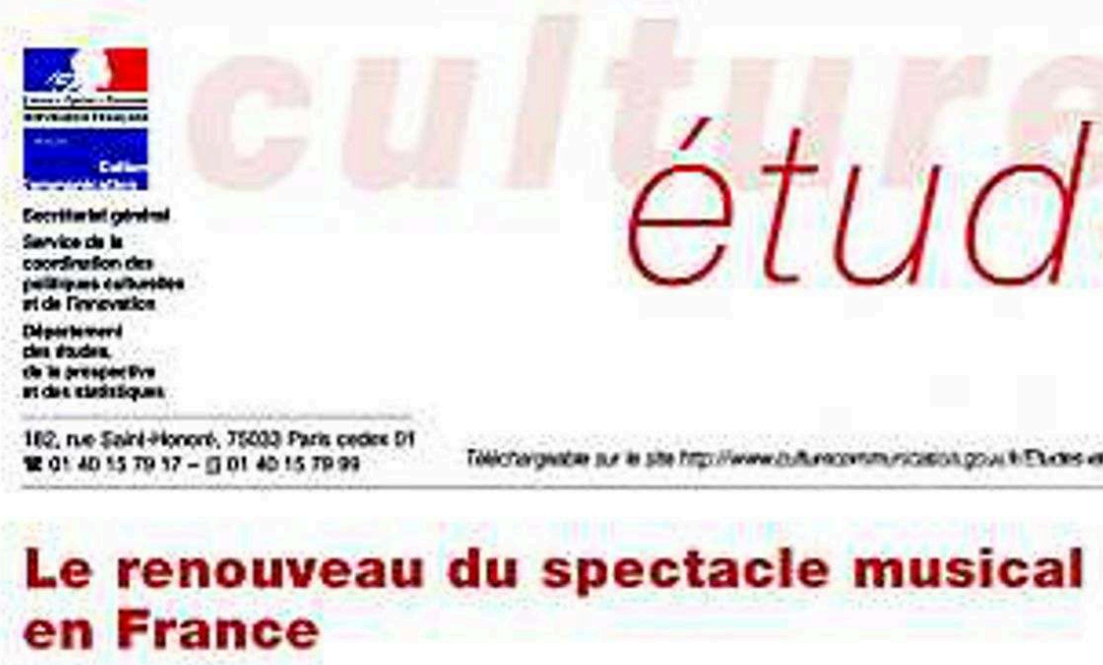

Xavier Dupuis et Bertrand Labarre*

The Resurgence of Musical Theatre in France

Dans le moede da spectixcle, Teppellision de - coens. de mesitak * a longsemps et considéné sorme pojo-

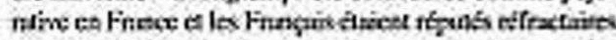
Leelte esthtique focks trop antio-savone. Fourtark ks siucts ble 11 sitne copognok el allemande ont demoater

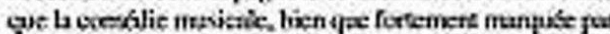
1. modele anglosason, peut trouver son public auss becn is Neu Yoek. Londres, Madrid ou Berlin qu' 3 S6oul

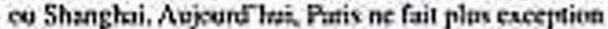
L cambitc muskate senthle avodr enta acquis ses lemes Ee reblesse en Frnoce et le zombre de spectacks musi. cunx a Jisficke ne cesse to croltre. Defer 2013, on

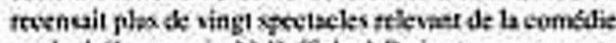
ol dh thefiere musical b tiffiche a Paris et on en compoe

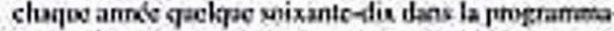

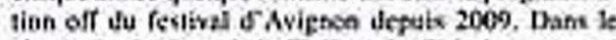
chamin intiestionnel, Is Thosire do Chiscket a ronood awos u Irikftice histrique \&e geozrammalcur de pikest

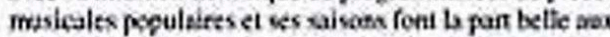
frumls clossiques de la comdie mosicale andricaine.

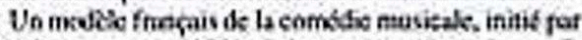

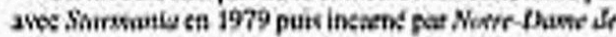

Turis en 1998 ( 3 millscas de spectateurs, 85 milibocs de

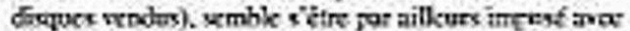

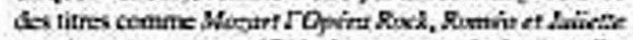
et. plus rocemment. $17 \times 9$, les usnumes de ia Bassiz. Enfin, ke metat en 2005 du bestre Mogader par Stage

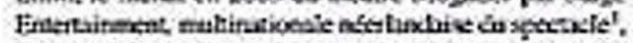
s'est troduit par l'apporition sur la sctac purisenox du

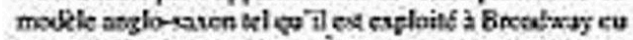

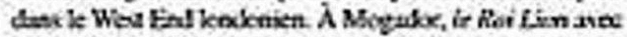
trois sisons, te 2007 a 2010 , a ains enregiste 1,5 mit. fics d" entrtes et obecrou trois fistinctions azs Molienes.

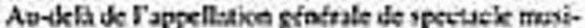
cal, trois syper de spoctucles ceceespendan a trois modeles teonomigues regonat sur des exipeders artio.

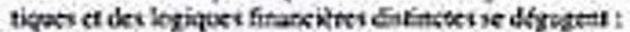
la combilic musicale it a fanceite, le theirse muical et

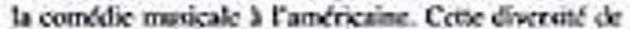

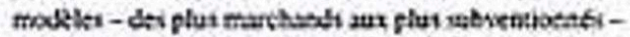
mootr, en pasuat, que ker eboix antistiques et keoco-

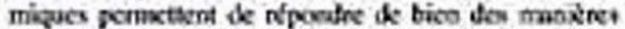

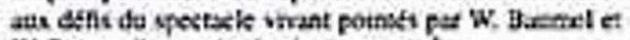
W. Bowen it y a pets de ciscquante ans?

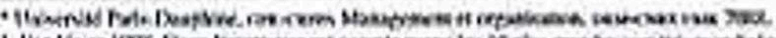

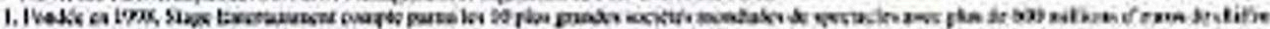

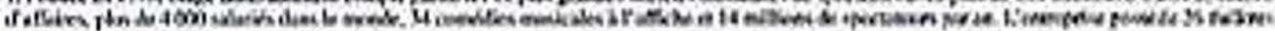

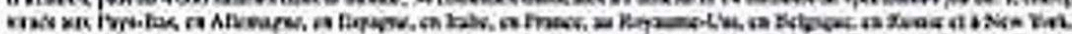

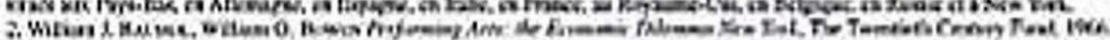




\section{Le renouveau du spectacle musical en France}

The Resurgence of Musical Theatre in France

\section{Xavier Dupuis et Bertrand Labarre}

Éditeur : Département des études, de la prospective et des statistiques

Lieu d'édition : Paris

Année d'édition : 2013

Date de mise en ligne : 8 juillet 2015

Collection : Culture études

ISBN électronique : 9782111399204

\section{Qboooks}

http://books.openedition.org

\section{Édition imprimée}

Date de publication : 1 novembre 2013

Nombre de pages : 12

\section{Référence électronique}

DUPUIS, Xavier ; LABARRE, Bertrand. Le renouveau du spectacle musical en France. Nouvelle édition [en ligne]. Paris : Département des études, de la prospective et des statistiques, 2013 (généré le 25 avril 2021). Disponible sur Internet : <http://books.openedition.org/deps/139>. ISBN : 9782111399204.

(C) Département des études, de la prospective et des statistiques, 2013

Creative Commons - Attribution - Pas d'Utilisation Commerciale 3.0 non transposé - CC BY-NC 3.0 

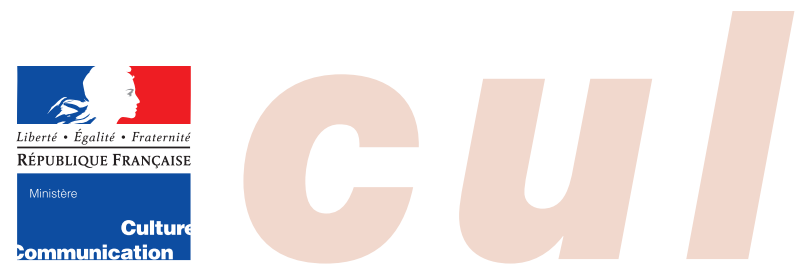

Secrétariat général

Service de la

coordination des

politiques culturelles

et de l'innovation

Département

des études,

de la prospective

et des statistiques

\section{Le renouveau du spectacle musical en France}

\section{Xavier Dupuis et Bertrand Labarre*}

\section{The Resurgence of Musical Theatre in France}

Dans le monde du spectacle, l'appellation de « comédie musicale » a longtemps été considérée comme péjorative en France et les Français étaient réputés réfractaires à cette esthétique jugée trop anglo-saxonne. Pourtant, les succès de la scène espagnole et allemande ont démontré que la comédie musicale, bien que fortement marquée par le modèle anglo-saxon, peut trouver son public aussi bien à New York, Londres, Madrid ou Berlin qu'à Séoul ou Shanghai. Aujourd'hui, Paris ne fait plus exception : la comédie musicale semble avoir enfin acquis ses lettres de noblesse en France et le nombre de spectacles musicaux à l'affiche ne cesse de croître. Début 2013, on recensait plus de vingt spectacles relevant de la comédie ou du théâtre musical à l'affiche à Paris et on en compte chaque année quelque soixante-dix dans la programmation off du festival d'Avignon depuis 2009. Dans le champ institutionnel, le Théâtre du Châtelet a renoué avec sa tradition historique de programmateur de pièces musicales populaires et ses saisons font la part belle aux grands classiques de la comédie musicale américaine.

Un modèle français de la comédie musicale, initié par avec Starmania en 1979 puis incarné par Notre-Dame de
Paris en 1998 (3 millions de spectateurs, 8,5 millions de disques vendus), semble s'être par ailleurs imposé avec des titres comme Mozart l'Opéra Rock, Roméo et Juliette et, plus récemment, 1789, les amants de la Bastille. Enfin, le rachat en 2005 du théâtre Mogador par Stage Entertainment, multinationale néerlandaise du spectacle ${ }^{1}$, s'est traduit par l'apparition sur la scène parisienne du modèle anglo-saxon tel qu'il est exploité à Broadway ou dans le West End londonien. À Mogador, le Roi Lion avec trois saisons, de 2007 à 2010, a ainsi enregistré 1,3 million d'entrées et obtenu trois distinctions aux Molières.

Au-delà de l'appellation générale de spectacle musical, trois types de spectacles correspondant à trois modèles économiques reposant sur des exigences artistiques et des logiques financières distinctes se dégagent : la comédie musicale à la française, le théâtre musical et la comédie musicale à l'américaine. Cette diversité de modèles - des plus marchands aux plus subventionnés montre, en passant, que les choix artistiques et économiques permettent de répondre de bien des manières aux défis du spectacle vivant pointés par W. Baumol et $\mathrm{W}$. Bowen il y a près de cinquante ans ${ }^{2}$.

\footnotetext{
* Université Paris-Dauphine, GFR-CREPA Management et organisation, DRM-CNRS UMR 7088.

1. Fondée en 1998, Stage Entertainment compte parmi les 10 plus grandes sociétés mondiales de spectacles avec plus de 600 millions d'euros de chiffre d'affaires, plus de 4000 salariés dans le monde, 34 comédies musicales à l'affiche et 14 millions de spectateurs par an. L'entreprise possède 26 théâtres situés aux Pays-Bas, en Allemagne, en Espagne, en Italie, en France, au Royaume-Uni, en Belgique, en Russie et à New York.

2. William J. BaumoL, William G. Bowen Performing Arts: the Economic Dilemma New York, The Twentieth Century Fund, 1966.
} 
DES DONNÉES ÉCONOMIQUES

ET DE FRÉQUENTATION ENCORE

LACUNAIRES EN RAISON D'UNE RECONNAISSANCE TARDIVE DU GENRE

\section{Des sources lacunaires pour appréhender l'activité et la fréquentation des spectacles}

À la différence de la ligue de Broadway League, organisation professionnelle qui rassemble 40 théâtres de ce quartier de New York et communique de façon transparente sur les entrées du box-office y compris pour les théâtres du Off Broadway, ou encore de la Society Of London Theater (SOLT) qui fédère les producteurs, propriétaires et directeurs des grands théâtres commerciaux et subventionnés du centre de Londres (assimilé au quartier du West End), il n'y a pas d'organisme unique qui comptabilise les entrées des spectacles musicaux en France. En effet, la taxe fiscale sur les spectacles de variétés (3,5\% de l'assiette) à laquelle sont assujetties les représentations de spectacle vivant est collectée soit par le Centre national des variétés et du jazz (CNV), soit par l'Association pour le soutien du théâtre privé (ASTP). C'est la nature ou le contenu d'un spectacle qui détermine son rattachement à l'un ou l'autre des organismes collecteurs, et non le critère du lieu de représentation. Le législateur a en effet pris le parti de distinguer deux esthétiques : l'une relève de la variété et l'autre de l'art dramatique, lyrique et chorégraphique privé. Autant dire que la frontière n'est pas toujours évidente, d'autant que le décret $\mathrm{n}^{\circ}$ 2004-117 du 4 février 2004 énumérant les spectacles de variétés fait état de «certains spectacles musicaux ou comédies musicales », expression qui ne s'interprète pas aisément. Les litiges de compétence entre les deux organismes se sont multipliés dans la période récente, requérant la réunion régulière d'une commission d'arbitrage sous la tutelle du ministère de la Culture et de la Communication. De 2009 à 2011, 15 arbitrages ont été rendus, dont 7 favorables à l'ASTP et 8 au CNV. Beaucoup de spectacles, dont ceux produits par Stage Entertainment, échappent au CNV (Mogador est membre de l'ASTP) et donnent lieu à des litiges récurrents. L'enjeu financier est de taille: les arbitrages rendus en faveur de l'ASTP pour les comédies musicales le Roi Lion et Zorro, programmées aux Folies Bergères par Stage Entertainment, lui ont ainsi permis de percevoir 1,475 million d'euros de taxe entre 2008 et 2010, soit un tiers des recettes de taxe perçues par l'ASTP au cours de ces trois années. Cette rivalité entre organismes collecteurs explique en partie la difficulté à réunir des données relatives à la production de spectacles musicaux en France, d'autant que l'ASTP ne publie pas de statistiques annuelles de fréquentation ventilées selon le genre de spectacles.

\section{Un secteur concentré}

En 2011, la région Île-de-France concentrait $55 \%$ des représentations et $43 \%$ des entrées pour les spectacles assujettis à la taxe perçue par le CNV (hors Mama Mia! donné au théâtre Mogador, dont les données de fréquentation ne sont pas disponibles). À la concentration géographique s'ajoute une concentration des revenus. En 2011, les 20 premiers spectacles musicaux déclarés au CNV représentaient $97 \%$ des recettes générées par l'ensemble des spectacles musicaux. La fréquentation est concentrée sur quelques titres donnés dans des lieux à grande jauge qui peuvent faire varier les données de fréquentation de façon parfois spectaculaire. Ainsi en 2005, le Roi Soleil assurait à lui seul, en trois mois d'exploitation parisienne, $54 \%$ de la taxe versée par cette esthétique et $49 \%$ des entrées et, en 2007 , avec une tournée puis un retour à Paris, $67 \%$ de la taxe et $59 \%$ des entrées, totalisant à lui seul 1,7 million de spectateurs.

Enfin, la fréquentation est concentrée dans le temps, les week-ends et en fin d'année. La comédie musicale est nettement surreprésentée parmi les spectacles familiaux et $70 \%$ des représentations sont programmées en fin de semaine, tandis que les spectacles programmés le mercredi ne représentent que $11 \%$ de la fréquentation hebdomadaire, signe que les programmateurs n'en font pas un genre favori pour le jeune public. En termes de saisonnalité, la comédie musicale figure en bonne place parmi les spectacles de fin d'année : près de $20 \%$ des représentations sont programmées en décembre (contre $8 \%$ tous genres confondus) et rassemble $22 \%$ de la fréquentation payante de l'année (contre $9 \%$ tous genres confondus).

\section{Modestie de l'activité française...}

La Fédération du musical estimait, pour l'année 2006, le chiffre d'affaires global du genre à quelque 40 à 50 millions d'euros, hors produits dérivés. Depuis cette date, le nombre de spectacles musicaux à l'affiche a progressé avec l'installation au théâtre Mogador de Stage Entertainment. Dans l'ensemble des spectacles dont l'activité est suivie par le CNV, avec 1100 représentations, les comédies musicales ne représentaient, en 2011, que $2 \%$ du nombre de représentations mais $5 \%$ de la fréquentation totale des représentations payantes et $6 \%$ de l'assiette déclarée avec un prix moyen du billet de 40 euros, soit 40 millions d'euros de recette annuelle de billetterie. La programmation d'un ou deux grands spectacles peut entraîner une variation importante des recettes : en 2009 et 2010, les spectacles assujettis à la taxe perçue par le CNV avaient généré une assiette annuelle de l'ordre de 47 millions. On peut estimer le chiffre d'affaires du spectacle musical à environ 60 à 70 millions en 2013, un chiffre non négligeable qui reste cependant très en deçà des chiffres d'affaires américain, britannique et même allemand. 


\section{L'importance du musical aux États-Unis, au Royaume-Uni et en Allemagne en quelques chiffres}

Aux États-Unis, au cours de la saison 2011-2012, avec 12,3 millions de billets vendus au prix moyen de 95 dollars, les recettes brutes de billetterie des 40 théâtres de la Broadway League ont atteint 1,158 milliard de dollars dont 934 millions pour les seuls spectacles musicaux (hors recettes des théâtres Off Broadway). De 2000 à 2010, la recette globale des théâtres de la ligue a progressé de $53 \%$. Pour la seule semaine du 7 au 14 janvier 2013, une semaine traditionnellement difficile après les fêtes de fin d'année, Broadway a réalisé 17,8 millions de dollars de recettes avec 24 spectacles à l'affiche, 192 représentations et 187700 spectateurs.

À ces données de fréquentation s'ajoutent les résultats des spectacles en tournée qui sont tout aussi étonnants : au cours de la saison 20092010, 40 spectacles en tournée dans 190 villes, 9900 représentations, 15,9 millions de billets (soit une jauge moyenne de 1000 places par représentation) et une recette de 947 millions de dollars, pour un prix moyen unitaire du billet de l'ordre de 60 dollars. En dix ans, la recette des tournées a presque doublé (+ $80 \%$ ). La ligue estime ainsi qu'au cours de la saison 2008-2009, Broadway a contribué à l'économie newyorkaise pour 11 milliards de dollars et représenté 86000 emplois tandis que les tournées généraient un impact de 3,35 milliards de dollars se répartissant entre les villes accueillant les spectacles en tournée pour près de 3 milliards et la ville de New York pour 400 millions.

Au Royaume-Uni, le West End attire encore plus de spectateurs que Broadway, avec quelque 14 millions de billets vendus en 2012. Selon la Society of London Theater, qui fédère les producteurs, les propriétaires et les directeurs des grands théâtres commerciaux ( $85 \%$ des membres) mais aussi subventionnés (comme le Royal Opera House of Covent Garden) du centre de Londres, assimilé au quartier du West End, les recettes théâtrales de l'année calendaire 2012 se sont établies à 529,8 millions de livres dont 322,2 millions de livres (soit $65 \%$ ) pour les seuls spectacles musicaux. La Solt estime à 2 milliards de livres et 41000 emplois le poids du théâtre londonien dans l'économie britannique. Pour la seule ville de Londres, sans West End, ce sont chaque soir 15000 tables dans les restaurants (70\% des spectateurs y dînent) et 5000 chambres d'hôtel ( $15 \%$ des spectateurs y logent) qui seraient vides.

Enfin, en Allemagne, selon la société fédérale des industries du spectacle ${ }^{1}$, les Allemands ont dépensé 2,2 milliards d'euros en spectacles de musique en 2009, tous genres confondus. En 2003, les seuls spectacles musicaux représentaient $19 \%$ du marché (soit quelque 500 millions d'euros) devançant l'opéra (15\%), la variété internationale (14\%), le concert classique (13\%) et enfin le cabaret (11\%).

1. Konsumentenstudie 2009, IDKV-Bundesverband der Veranstaltungswirtschaft e.V.

\section{... malgré un public acquis}

Le spectacle musical relève pour l'essentiel de l'économie non subventionnée. Le genre est massivement produit par des structures privées ayant un statut juridique commercial $(82 \%$ des structures déclarantes auprès du $\mathrm{CNV})$, tandis que la part du secteur associatif ( $8 \%$ ) et des structures publiques $(9 \%)$ reste marginale. Le spectacle musical ne peut donc pas exister sans un public nombreux et fidèle.

En 2011, tirés par Mozart l'Opéra Rock, Dracula ou encore la Mélodie du bonheur et Sweeney Todd (deux spectacles du Théâtre du Châtelet), les spectacles musicaux ont rassemblé 1,1 million d'entrées, soit $5 \%$ de la fréquentation totale comptabilisée par le CNV contre 1,3 million d'entrées en 2010 et 1,4 en 2009. Ces fortes variations d'une année sur l'autre s'expliquent par l'effet blockbuster de certains spectacles. En estimant que les entrées comptabilisées par l'ASTP - dont celles des spectacles présentés à Mogador par Stage Entertainment représentent plus d'un demi-million de spectateurs, on peut raisonnablement évaluer le nombre annuel d'entrées totales à près de 2 millions en 2011. Avec une fréquentation plus faible que les spectacles de chanson (5,2 millions) et les spectacles de pop rock et assimilés (4,6 millions), mais qui dépasse celle du jazz et des musiques improvisées (1,5 million) et des spectacles de musiques du monde (1,2 million), le spectacle musical a donc su trouver un public, même s'il est bien plus restreint que celui de West End (14 millions de billets en 2012) et de Broadway (12,3 millions en 2011-2012). Le marché français n'est évidemment en rien comparable au marché américain auquel la célébrité mondiale de Broadway confère une attractivité exceptionnelle : à New York, près des deux tiers des billets sont achetés par des touristes (63\% au cours de la saison 2011-2012). Mais, plus encore, c'est l'existence d'un public pour lequel le spectacle musical participe des références culturelles qui explique l'écart entre la France et le monde anglo-saxon. Le spectateur moyen de Broadway est âgé de 43 ans et assiste à 4 spectacles par an. Les femmes représentent $67 \%$ du public new-yorkais et $72 \%$ de celui des spectacles en tournée. À Broadway, $38 \%$ des spectateurs sont titulaires d'un diplôme de l'enseignement supérieur. Leur revenu annuel moyen s'élevait, en 2012, à 193800 dollars (contre quelque 38000 dollars pour l'ensemble de la population américaine). $43 \%$ des spectateurs disposaient ainsi d'un revenu annuel d'au moins 100000 dollars (contre $22 \%$ pour l'ensemble de la population américaine). Le groupe des assidus, c'est-à-dire ceux qui assistent à 15 représentations au moins par an, représentait $5 \%$ du public de Broadway et $29 \%$ de l'ensemble de la fréquentation au cours de la saison 20112012. Quant au public des tournées américaines, il assiste en moyenne à 6 spectacles par an et est composé pour près de la moitié d'abonnés (44\%). 


\section{TROIS ESTHÉTIQUES POUR UN MÊME NOM : LE SPECTACLE MUSICAL}

On appelle spectacle musical tout type de spectacle associant plusieurs artistes dans le cadre d'une production, présentant une trame narrative mise en scène et en musique. Le champ ainsi défini s'étend donc de l'opéra au concert et au one-man-show; en sont exclus les spectacles relevant clairement des variétés, et recensés comme tels par le Centre national de la variété, de la chanson et du jazz (CNV), de même que les revues, telles celles proposées au Lido ou au Moulin Rouge. L'ensemble ainsi défini reste très hétérogène d'un point de vue esthétique, organisationnel ou encore économique. On y trouve des spectacles de toutes dimensions: beaucoup de petites formes mais aussi de grandes productions présentées dans des salles de type palais des sports. Héritées de la comédie musicale à l'anglo-saxonne et de l'opérette française, trois formes de spectacles musicaux qui s'opposent sur leurs fondements esthétiques renvoient également à des modèles économiques très différents : la comédie musicale à la française, le théâtre musical et le spectacle musical à l'anglo-saxonne.

\section{La comédie musicale à la française, un modèle proche du spectacle de variétés}

\section{L'histoire du genre}

Son œuvre abondante a fait de Jacques Offenbach la référence majeure du théâtre chanté et de l'opéra-bouffe. Mais, plus encore, il a été le point d'ancrage du modèle historique de l'opérette, dépassant en cela Hervé qui avait véritablement initié le genre et l'avait exporté à Londres. L'opérette s'affirme en France au début du $\mathrm{XX}^{\mathrm{e}}$ siècle et attire de grands compositeurs, au moment où se développent également le music-hall et la revue, un genre auquel Paris devra bientôt une partie de sa notoriété mondiale avec des lieux comme le Lido, le Moulin Rouge ou les Folies Bergères. Profitant de l'engouement des publics, des diffuseurs, des auteurs compositeurs et des artistes de scène, une nouvelle esthétique s'ébauche, reposant sur les chansons, qui s'insèrent dans une trame dramatique et un livret mais peuvent également exister par elles-mêmes. Conçues comme telles, elles pourront par la suite s'insérer dans des concerts, des tours de chant, faire l'objet d'une édition sur disque. Fin 1945, Francis Lopez, déjà auteur fameux de chansons, écrit rapidement sa première opérette, sur un livret de Raymond Vincy, La Belle de Cadix, et engage un ténor inconnu, Luis Mariano. C'est le point de départ d'une collaboration en trio où le librettiste, l'interprète masculin principal et le compositeur vont dominer largement la scène française du théâtre chanté, avec une opérette écrite et produite par an.

L'arrivée de la pop, du rock et l'ouverture de la période yé-yé sont fatales à l'opérette et la crise est évidente au début des années 1970 : public vieillissant et connoté populaire, dévalorisation du genre, budgets déclinants alors que le genre est onéreux, tournées en province qui achèvent l'esthétique originelle par une insuffisance de décors, de machinerie et de costumes... Sans le soutien public et celui de quelques rares maisons d'opéras intégrant la survie du genre dans leur ligne artistique, l'opérette aurait disparu. Le Châtelet, véritable temple de l'opérette, repris par la mairie de Paris et baptisé Théâtre musical de Paris, se voit confier une mission qui le placera, jusqu'à l'arrivée de Jean-Luc Choplin en 2005, en concurrent direct de l'Opéra national de Paris. Par ailleurs, la réorientation chaotique de Mogador et la fermeture de la Gaîté Lyrique ôtent finalement à l'opérette tout espoir de survie sur les grandes scènes parisiennes. La France peu à peu perd le goût de la création dans le théâtre musical, ce qui explique la difficulté à relancer le genre dans le pays.

En 1974, la Révolution française est la première comédie musicale à grand spectacle à émerger écrite par Alain Boublil et Claude-Michel Schönberg. Le projet, d'abord conçu comme un double album de chansons, connaît un tel succès qu'il est monté en comédie musicale au palais des sports, posant les jalons de l'esthétique à la française, repris de façon plus méthodique encore par Starmania en 1979: un disque pivot du spectacle, des tableaux mis en scène sur les chansons, une absence notable de récitatifs, un spectacle programmé dans de très grandes salles pour une série limitée de dates. Mais le genre se développe timidement. Le monde de la variété s'empare d'un domaine susceptible de lui faire vendre des disques mais la France ne parvient pas à s'approprier l'esthétique en pleine évolution ailleurs. Pourtant, il y a un public: les Misérables, par exemple, ont rassemblé 500000 spectateurs en France sur 107 représentations, la Révolution française, 300000 et Starmania initialement programmé pour 15 dates au Palais des congrès de Paris, lance les carrières de presque tous ses interprètes et vend pour un nombre d'albums record. Les multiples reprises rassembleront 5 millions de spectateurs. Pourtant, la France souffre alors d'un déficit de vivier, de structures spécialisées, d'un milieu professionnalisant : le spectacle musical est traversé de façon éphémère par des vedettes de la mise en scène de théâtre ou de la chanson qui, dans le meilleur des cas, y réalisent des événements sans lendemain. C'est donc après une longue période de désintérêt que le genre se renouvelle quand, en 1998, Notre-Dame de Paris, porté par les ventes du disque, rencontre un succès sans précédent avec 3,5 millions de spectateurs. Encouragés par cette percée, plusieurs producteurs phonographiques et audiovisuels tentent de dupliquer le phénomène et la décennie 2000 sera féconde pour cette esthétique. Parmi les succès, on peut citer les Dix Commandements (2000), Roméo et Juliette (2001), le Roi Soleil (2005), Mozart, l'opéra rock (2009), même si nombre d'autres productions ne séduiront guère le public.

Afin de limiter les risques encourus par la production, les sujets sont issus du répertoire classique romanesque ou théâtral, du patrimoine universel, d'événements historiques. Mais, en l'absence de règles et de conventions claires, l'esthétique n'est pas codifiée : l'orchestre est préenregistré, parfois les interprètes chantent en play-back. 
Les grosses productions se jouent dans de vastes salles aux jauges dépassant 4000 places, qui tiennent les spectateurs éloignés de la scène et donc de l'action dramatique, ce qui conduit les grandes comédies musicales à la française à la surenchère en terme de spectaculaire (pour Mozart, l'Opéra Rock on comptait 12 décors et 400 costumes). Elles renoncent ainsi à l'intimité avec les spectateurs et les effets de mise en scène l'emportent sur la trame dramaturgique. Les ballets existent... ou non, les chansons, conçues pour être entendues à la radio, peuvent parfaitement s'écouter hors du contexte du spectacle dont elles sont pourtant tirées; enfin, les livrets sont redoutablement dépourvus d'humour. La tradition française de l'opérette légère et de l'opéra-bouffe savait pourtant manier l'humour avec talent et les Anglo-Saxons y excellent aujourd'hui, nullement effrayés que le rire constitue le contrepoint au propos initial.

\section{Des succès qui s'appuient sur une communication médiatique savamment orchestrée}

Starmania, Notre-Dame de Paris, les Dix Commandements... : ces succès populaires et médiatiques suggèrent qu'il existe un modèle de création de grand spectacle musical à la française dominant en France. Il constitue de fait une spécificité française et rares sont les titres qui ont rencontré le succès à l'étranger. Fondé sur une esthétique particulière très éloignée du modèle anglo-saxon, ce type de comédie musicale, particulièrement coûteux puisque les budgets se comptent en millions d'euros, est construit comme un tour de variétés ou de rock. Les auteurs et les artistes de plateau viennent d'ailleurs du monde de la scène de variétés. Le genre accorde ainsi une primauté absolue à la chanson formatée pour devenir un succès à la radio car toute l'économie du grand spectacle musical à la française repose sur l'exploitation discographique. Le spectacle vivant est ici un produit complémentaire et le livret a pour but de donner du sens à 10 à 15 chansons en les reliant par un fil rouge susceptible d'intéresser le plus grand nombre. Les titres peuvent aisément s'inscrire dans diverses histoires et ne participent pas particulièrement au développement dramaturgique. Le spectacle est une vitrine, un produit d'appel pour vendre le disque. La logique est très risquée car lorsque le disque ne séduit pas, c'est tout le modèle qui s'effondre. Si l'enregistrement n'est pas d'abord un bon produit discographique, apprécié en radio, le projet est condamné. C'est pourquoi le modèle a besoin d'un appui médiatique très puissant pour vendre le disque et remplir les salles dans lesquelles le spectacle va être donné. Les jauges, comprises entre 3500 et 7000 places, à Paris comme en tournée, nécessitent de créer en amont une notoriété qui s'appuie sur des partenariats avec les médias, ce qui explique que l'on trouve souvent des groupes comme Amaury ou Lagardère dans le plan de financement. L'imbrication stratégique entre production discographique et production scénique réclame un planning parfaitement maîtrisé pour coordonner la sortie des différents produits audiovisuels et les dates clés de la tournée.

\section{Mozart l'opéra rock: un planning médiatique exemplaire}

- septembre 2008 : affichage dans le métro, simple appel à la curiosité (teasing) car l'affiche est énigmatique. Ouverture de la billetterie. - décembre 2008 : Warner choisit la chanson qui doit être lancée en premier. La présence médiatique s'accroît.

-janvier 2009 : sortie sur les ondes du premier single, puis en téléchargement payant. Le CD contient également un clip et une vidéo du tournage (making-of) (numéro 1 des ventes physiques pendant 5 semaines, il est resté 11 semaines dans le top 5).

-16 mars : sortie du deuxième titre single.

-27 avril : sortie de l'album simple, composé de 12 titres.

-22 juin : sortie du troisième single.

-21 septembre : sortie de l'album intégral, composé de 27 titres.

-22 septembre : première du spectacle au Palais des Sports de Paris (à l'affiche jusqu'au 3 janvier 2010).

-9 novembre : sortie du quatrième single.

-26 novembre : sortie de l'album intégral édition de luxe, composé de 27 titres, d'un DVD avec des bonus (coulisses du spectacle, clips, etc.). - du 4 février au 3 juillet 2010 : tournée en France, Belgique et Suisse.

- mars 2010 : sortie d'un nouveau single.

- novembre 2010 : sortie du DVD du spectacle (quelque 600000 exemplaires mis en place dans les circuits de distribution).

- du 9 novembre au 16 décembre 2010 : retour de la production au Palais des sports de Paris.

L'internet est largement utilisé pour accompagner ce plan de communication: site officiel, forum, blog, groupe de 140000 internautes sur Facebook... Classé numéro 1 des ventes de spectacles en 2009-2010 avec plus de 800000 billets vendus, Mozart l'opéra rock est un immense succès. L'articulation entre sortie du disque et présentation du spectacle a permis de développer une véritable synergie : l'album sert à vendre des billets puis le spectacle et la pression médiatique qu'il génère (télévisions, radios, Internet) dopent les ventes de disques et de DVD.

Classé au premier rang des investissements publicitaires dans le domaine du disque en 2009, avec 6,11 millions d'euros, dont 4,88 investis par Warner Music en télévision (majoritairement sur TF1, coproducteur) et 1,23 million d'euros investis en radio à hauteur de $39 \%$ sur Virgin Radio (également coproducteur), $36 \%$ sur NRJ, $21 \%$ sur RFM, soit majoritairement sur les radios musicales, dites de format jeune, Mozart l'opéra Rock a été le $5^{\mathrm{e}}$ des titres les plus diffusés, avec 27280 diffusions. Une stratégie qui a porté ses fruits puisque, avec plus de 580000 ventes physiques et numériques en 2009 (pour les albums et les différents singles), Mozart l'opéra Rock s'est placé au $10^{\mathrm{e}}$ rang des ventes du top 50. 
Les producteurs de ce type de spectacle sont au nombre de quatre - Charles Talar, Jean-Claude Camus, Dove Attia et Albert Cohen - et constituent un véritable oligopole. Incontournables par leur professionnalisme, dotés de structures de production adaptées, ils sont capables de mobiliser des fonds conséquents au profit de la comédie musicale à la française. La production de ces spectacles s'appuie cependant sur des structures qui demeurent modestes par rapport aux sommes en jeu. Confrontés à des montages financiers complexes associant des groupes multimédias ou des multinationales du spectacle, tout l'enjeu tient à leur capacité à travailler dans le court terme car les capitaux doivent garder une liquidité élevée. Aussi, le projet s'étend sur 36 mois environ, avec des décaissements effectifs sur un an et les spectacles français sont d'abord pensés pour le public français. Avec un investissement autour de 8 à 9 millions d'euros hors les droits pour une première exploitation, soit au total quelque 10 millions d'euros, les producteurs ont une exigence de retour sur investissement à 3 mois, toutes exploitations confondues, la pérennité des revenus d'édition, plus ou moins importants, assurant éventuellement des profits à plus long terme. Le spectacle ne sera jamais remonté, sauf très rares exceptions. Cela ne laisse aucune pérennité aux œuvres qui n'existeront que le temps de leur amortissement et celui d'atteindre les profits escomptés : une première série de dates à Paris, puis dans les grandes villes de région et en pays francophones (Belgique et Suisse), enfin retour à Paris, soit de 12 à 18 mois.

Pour autant, toute l'économie du genre reposant sur le succès discographique, le modèle n'est-il pas condamné à disparaître, en dépit de réussites comme celle de Mozart, l'opéra Rock?

\section{De l'opérette au théâtre musical}

\section{Brève histoire du genre}

Une autre esthétique, en totale opposition avec la comédie musicale à la française, voit le jour en France au cours de la même période. Revendiquant leur rattachement au théâtre, de nouveaux créateurs, issus précisément du monde du théâtre et non pas de la variété, se concentrent sur l'écriture d'un livret solide, capable de résister à la représentation vivante : une démarche de librettiste exigeant sur la qualité littéraire mais cependant attaché à la musique, la chanson, l'expression dramatique et à leur mariage sur une scène. En cela, les fondements esthétiques de ce nouveau type de spectacle présentent des ressemblances avec le modèle anglo-saxon, mais il n'en va pas de même en termes de moyens : il s'agit le plus souvent de productions légères qui s'appuient sur des budgets modestes. Cette tendance créatrice traduit le souhait d'un certain nombre d'auteurs de recentrer la création française sur l'écriture de pièces de théâtre dans lesquelles on chante, on danse éventuellement et où l'on raconte une histoire en musique. Créatures d'Alexandre Bonstein en 1998, Chance! d'Hervé Devolder en 2000, qui a franchi le cap symbolique des 1000 représentations sur le territoire, Panique à Bord de Stéphane Laporte, prix
SACD en 2005, Camille $C$ de Jonathan Kerr, récompensé par un Molière en 2005, le Cabaret des hommes perdus de Patrick Laviosa et Christian Siméon, récompensé par un Molière en 2006 ou encore l'Opéra de Sarah d'Alain Marcel, Molière en 2008... : autant d'œuvres qui semblent presque fonder un genre. Leur approche se veut un retour à l'essentiel : aucun artifice technique, aucun dérivatif spectaculaire ne doit faire perdre de vue le cœur d'un spectacle musical, un livret qui raconte une « bonne histoire ». En cela, le théâtre musical est l'héritier direct de l'opérette. Les chansons font partie de la narration, elles forment la continuité dramatique et ne sont pas réduites à des respirations musicales. Ce théâtre, qui va de la très petite compagnie au rassemblement de capitaux plus lourds (mais très en deçà du niveau anglo-saxon), est en pleine effervescence. Des auteurs développent la création dans ce secteur qui est considéré en tant que tel : une catégorie à part entière, à la fois théâtrale et musicale et revendiquant également l'appellation de spectacle musical. Les spectacles destinés au jeune public y sont nombreux (Merlin l'Enchanteur, Alice au Pays des Merveilles, Scooby-Doo...). Quelques théâtres se sont particulièrement ouverts au cours de ces dernières années à ce type de spectacle : le théâtre municipal d'Oullins, le théâtre de la Croix-Rousse à Lyon, la Maison de la culture de Nevers. Mais l'essentiel de la production est réalisé à l'initiative de compagnies ou de producteurs dans le cadre de structures privées, peu ou pas subventionnées. Son économie est fragile et bien éloignée des grands spectacles anglo-saxons ou français.

\section{Un modèle économique proche de celui du spectacle vivant subventionné}

Le modèle économique est proche de celui du théâtre, celui des petites compagnies pour les productions les plus modestes, celui du théâtre privé pour les productions les plus ambitieuses. Une typologie des spectacles de théâtre musical peut être établie à partir des effectifs artistiques mobilisés et des budgets et identifie trois formats de spectacle. Le premier réunit en général un chanteur et un musicien pour un budget de 30000 à 50000 euros en montage. Le deuxième comprend quelques comédiens, chanteurs et un deuxième musicien pour un budget de l'ordre de 150000 à 200000 euros. Enfin, le troisième, plus ambitieux, compte 7 à 8 musiciens et 15 à 20 comédiens pour un budget d'environ 700000 euros, parfois davantage. Une grande partie de la production du théâtre musical - peu ou prou l'ensemble des très petits et des petits spectacles - appartient au paysage classique des compagnies de théâtre. Par beaucoup d'aspects, le théâtre musical rencontre les mêmes problèmes que l'ensemble du spectacle vivant : manque de moyens, recherche tous azimuts de subventions, recours massif à l'intermittence, manque de visibilité dans un marché où l'offre est foisonnante, lieux de diffusion difficiles d'accès, etc. Mais le théâtre musical présente des handicaps supplémentaires propres à sa démarche artistique et souffre d'un certain ostracisme des tenants de la culture légitime et de la réticence des programmateurs à se lancer dans sa diffusion. 
Si monter une pièce de théâtre traditionnel, un tour de chant, un ballet requiert un temps certain, monter une esthétique qui intègre artistiquement les trois genres en requiert davantage. Marier les genres suppose un temps de répétition long, des artistes polyvalents, la permanence des équipes et des plateaux artistiques plus lourds que dans le théâtre dramatique. À cela s'ajoutent des droits d'auteurs importants, les ayants droit étant nombreux : librettiste, compositeur, metteur en scène, chorégraphe, arrangeur, voire chef de chœurs ou maître de chant... Par ailleurs, une équipe lourde et donc coûteuse à déplacer, des prix de cessions forcément élevés et jugés prohibitifs par les programmateurs, des plateaux n'ayant pas toujours la taille et l'équipement adéquats font que la tournée, indispensable à la survie d'un spectacle, est bien difficile à organiser et ce, d'autant plus que les programmateurs de salles en région restent réticents à programmer cette esthétique.

Étant donné le nombre de compagnies de spectacle vivant prétendant émarger à des volumes d'aides publiques décroissantes, il est devenu de plus en plus difficile aux directeurs de troupe et à leur producteur, de trouver les éléments du montage financier qui garantisse la création. Une résidence est souvent le point de départ pour réunir l'équipe et organiser les répétitions dans des conditions matérielles suffisantes. Lorsqu'un théâtre accepte d'accueillir une troupe pour une création, voire de la prendre en résidence rémunérée pour ensuite la coproduire, la résidence ne prend sens que si une tournée ou du moins une série de dates est prévue (30 à Paris, 15 sinon en région au minimum), une condition à l'obtention de nombreuses aides. Plusieurs sociétés d'auteurs peuvent soutenir le répertoire du spectacle musical (Spedidam, Arcadi, Sacem, SACD) mais sa pluridisciplinarité peut se retourner contre lui. Ainsi un spectacle musical théâtral peut-il être renvoyé par la SACD vers la Sacem en raison d'une composante musicale élevée, surtout si les chansons peuvent donner lieu à une exploitation phonographique. La Sacem à son tour n'hésitera pas à renvoyer vers la SACD parce que la présence d'un livret et d'une continuité dramatique jouée sur scène, fut-elle construite par des chansons, fait entrer l'œuvre dans la catégorie du théâtre.

Les producteurs ont donc bien du mal à atteindre la taille critique qui permet de mobiliser des capitaux en quantité suffisante pour mener à bien des projets ambitieux. L'économie du théâtre musical reste extrêmement fragile et, bien souvent, condamne les projets à des choix drastiques : réduction de l'orchestre à un ou deux instruments ou recours à une bande enregistrée... Sur le segment du théâtre musical, ces dernières années ont pourtant vu émerger quelques producteurs - Serge Tapierman (Un Violon sur le toit, Grease), le producteur italien Lorenzo Vitali (Fame, Hair) assisté en France par Marco Daverio, ou encore Pascal Bernardin - dont le dernier en date affiche certaines ambitions, comme en atteste sa dénomination (French West End, société créée en 2012 par Gilles Ganzmann, producteur télévisuel). En général, ils se portent acquéreurs de droits d'exploitation de spectacles préexistants dont l'image et la notoriété garantis- sent l'adhésion du public (The Full Monty, Grease, Un violon sur le toit...). Ils développent peu la création originale mais s'attachent en revanche les talents d'équipes locales sur le plateau comme à la mise en scène.

\section{Une greffe réussie? Quand le spectacle musical anglo-saxon s'installe à Paris au théâtre Mogador}

\section{La comédie musicale à l'américaine : brève histoire}

La comédie musicale américaine est née de l'union de deux genres typiquement britanniques : le burlesque et la revue de music-hall dénommée aux États-Unis « vaudeville». Le premier apparaît vers 1830 en GrandeBretagne. Il s'agit alors d'une petite revue plus ou moins construite autour d'une trame très légère. La revue de music-hall voit le jour une vingtaine d'années plus tard, dans les grands caf' conc' de Londres qui en compte près de 300 à la fin des années 1870. Ces deux genres jouissent, dès la fin du XIX ${ }^{\mathrm{e}}$ siècle, d'une grande popularité aux États-Unis et donnent naissance, au début du $\mathrm{Xx}^{\mathrm{e}}$ siècle, à une nouvelle esthétique, la comédie musicale américaine. Le développement rapide d'Hollywood et de Times Square, le quartier des théâtres de Broadway à New York, est à l'origine de l'industrie du divertissement, l'Entertainment Industry, qui a pour objectif la distraction du public. Tandis que l'opéra concerne l'élite et les pièces de théâtre issues du répertoire classique, la bourgeoisie, les salles populaires proposent des spectacles susceptibles d'être vus par la masse de migrants européens qui débarquent à New York sans parler l'anglais et recherchent des spectacles très visuels, simples dans leur livret, où la musique tient une place prépondérante. Au fil du temps, la comédie musicale américaine se théâtralise, en particulier avec l'arrivée du cinéma parlant et chantant, ses livrets sont de plus en plus construits et respectent la continuité dramatique. Cette nouvelle esthétique fait la part belle à la danse : une dimension qu'incarneront au cinéma Gene Kelly et Fred Astaire. La révolution du rock révélera à quel point le danseur a un statut particulier dans le monde du spectacle musical : les têtes d'affiche sont presque exclusivement des artistes hommes sachant danser, et Lisa Minelli et Barbra Streisand feront figure d'exceptions à Broadway, tandis que Judy Garland et Shirley Temple marqueront de leur côté le cinéma musical.

En 1957, West Side Story constitue le véritable tournant du Musical. Le triomphe de cette pièce écrite par Laurents et Sondheim et composée par Léonard Bernstein qui avait pourtant peiné à trouver le financement de son montage (musique trop « complexe», sujet de société trop contemporain, fin exagérément tragique aux yeux des producteurs), démultiplié par le film en 1961, ouvrit de nouvelles perspectives: on y dansait dans les parkings, la fin n'était pas un happy end, les costumes, l'univers et les personnages étaient issus du quotidien. Avec West Side Story, la comédie musicale s'inscrit dans le quotidien et le contemporain. S'ouvre alors 
une période de très forte créativité et de production prolifique. Apparaît un off Broadway, issu du mouvement hippie. S'installant dans des salles de petites jauges, des créateurs y développent des spectacles plus avant-gardistes voire des happenings musicaux. Le plus grand succès, Hair, en 1967, est récupéré par le On puis fait le tour du monde.

La scène anglaise, de son côté, n'est pas en reste. Au début des années 1970, un jeune compositeur, Andrew Lloyd Webber, qui deviendra quelques années plus tard un des producteurs incontournables du West End, parvient à renouveler le genre au moment où, aux ÉtatsUnis, la créativité est sacrifiée à la rentabilité. Webber triomphera ainsi avec Evita (1978), Cats (1981) ou encore The Phantom of the Opera à l'affiche de Broadway depuis le 26 janvier 1988 et totalisant plus de 10000 représentations.

Aujourd'hui, le spectacle musical anglo-saxon repose toujours sur des principes fondateurs, forgés par l'histoire du genre qui définissent une esthétique immédiatement identifiable par le public. Premier élément fondateur, le livret développe un sujet original ou l'adaptation d'une œuvre classique qui peut être traditionnelle (les Misérables de Boublil et Schönberg, montés en spectacle musical par Cameron Mackintosh en est le meilleur exemple) ou actualisée (Miss Saigon des mêmes auteurs est la modernisation pendant la guerre du Vietnam de Madame Butterfly de Puccini). Si certains succès cinématographiques se retrouvent sur scène (Martin Guerre, Spiderman...) le plus souvent, le film est dérivé du spectacle original (Mama Mia!, les Misérables...). Le cœur du spectacle est sa continuité dramaturgique et l'équilibre entre l'émotion, la profondeur des personnages et l'humour est essentiel. Même dans les romances flamboyantes (The Phantom of the Opera), les drames sociaux (Billy Eliott) ou les tragédies (West Side Story), ces spectacles offrent toujours des situations où la légèreté est à même de côtoyer la noirceur du propos général.

Deuxième élément fondateur du spectacle musical : un orchestre live. L'émotion créée par la musique jouée en direct est indispensable. Composé de trente musiciens pour les formations les plus nombreuses, l'orchestre est constitutif du spectacle, comme en témoigne le slogan de la grève qui paralysa Broadway en 2003 lorsqu'il fut question de réduire de 24 à 15 l'effectif moyen des orchestres: Don't spend real money for virtual music!

Troisième élément fondateur: le spectacle musical anglo-saxon est inséparable de la danse. Des chorégraphes comme Jérôme Robbins (On the Town, West Side Story), Michael Bennett (A Chorus Line) ou Bob Fosse (Dancin', Chicago) marquent de leur empreinte l'esthétique du genre. Il est impensable qu'un spectacle musical ne soit pas dansé, ce qui implique un autre élément fondateur: la polyvalence des artistes, capables aussi bien de chanter que de jouer et de danser.

Enfin, le Musical anglo-saxon tient à s'inscrire dans l'esthétique théâtrale : l'écrin du théâtre avec son lever de rideau, son architecture, son ambiance, son histoire. Cela peut paraître surprenant pour un art relativement récent qui ne craint pas le développement de la machinerie, des effets spéciaux. Autant de fondements à l'esthétique du Musical anglo-saxon que l'on ne retrouve pas dans la comédie musicale en France et qui s'oppose en tout point au virage vers le grand spectacle que le modèle français a pris dans le sillage de Starmania en 1979.

\section{Sa transposition en France au théâtre Modagor}

Le Musical anglo-saxon est une forme de spectacle qui a su constituer son économie dans le cadre du marché. Loin du stéréotype du produit artisanal, prototypique, non reproductible, qui est toujours associé au spectacle vivant, il s'est construit sur un mode quasiindustriel. Conçu d'emblée pour être joué le plus grand nombre de fois possible, il se situe donc à l'opposé du spectacle subventionné qui ne connaît qu'une série de représentations limitée, ce qui ne permet pas - sauf exception - un retour sur investissement. Pour pouvoir prétendre à la longue série, il convient néanmoins de réunir plusieurs conditions.

Il faut, en premier lieu, disposer d'une capacité financière permettant de supporter le risque économique et bénéficier d'un bassin de public suffisamment important pour remplir la salle plusieurs centaines de fois. Il faut ensuite faire des choix artistiques et disposer d'un lieu adapté proposant une jauge importante et une qualité d'accueil pour le public. Savoir communiquer et mettre en œuvre tous les outils du marketing est évidemment indispensable, de même que maîtriser les coûts de production, ce qui implique, pour le moins, des doubles distributions et une véritable gestion des ressources humaines. Enfin, il s'agit de maîtriser la chaîne de création de valeur (billetterie, spectacle, produits dérivés). Autant de conditions qu'il est difficile de réunir en France : ce modèle ne se trouve qu'à Paris, au théâtre Mogador, propriété de Stage Entertainment.

\section{Production et fréquentation : quelques repères}

Une œuvre reposant sur une dramaturgie, un orchestre qui joue en direct, une chorégraphie partie intégrante du spectacle, un théâtre à l'ancienne et une production soignée : l'esthétique du genre est par nature coûteuse. Â Broadway, les budgets d'investissement pour une nouvelle production atteignent couramment 20 millions de dollars (le record s'établissant à 65 millions pour Spiderman, Turn Off the Dark) et les coûts d'exploitation à la soirée peuvent approcher voire dépasser 100000 dollars. Si les budgets sont si élevés, c'est que, pour la création d'un nouvel ouvrage, un projet à Londres ou à New York s'élabore aisément sur 36 à 48 mois : 48 mois de production et de montage est le délai dont ont disposé, par exemple, Alain Boublil et Claude-Michel Schönberg pour Martin Guerre. Pour le montage d'une nouvelle production d'un ouvrage ayant déjà été créé (un «Revival»), les délais, bien que moindres, restent importants, de l'ordre de 24 mois. Aucun producteur français n'est en capacité financière de garantir une période de création aussi longue, tandis que les opérateurs de la comédie 
musicale à Broadway ou dans le West End sont des sociétés multinationales du spectacle: Cameron Mackintosh, le Really Useful Group d'Andrew Lloyd Weber, Disney Theatricals, Live Nation, et que rares sont les théâtres indépendants: parmi ses quarante théâtres, la Ligue de Broadway n'en répertorie que deux qui comptent parmi les plus petits. En revanche, Shubert Organization détient 17 théâtres, Nederlander Organization 9, et Jujamcyn Theatres 5. Disney ne possède qu'un seul théâtre, le New Amsterdam Theatre (1750 places), tout comme Live Nation (Foxwoods Theatre, 1810 places). Si le temps de préparation d'un spectacle est aussi long, c'est aussi parce que les producteurs recourent aux répétitions publiques payantes (previews), destinées à régler le spectacle mais aussi à le tester auprès du public. La production de Spider-man, Turn Off the Dark a ainsi commencé en répétition publique le 28 novembre 2010 pour ne connaitre sa première officielle que le 14 juin 2011, après quelque 200 répétitions publiques.

Pour devenir rentable, un spectacle doit, tout en couvrant les coûts de fonctionnement du théâtre, dégager une marge par représentation qui permettra dans un premier temps d'amortir le coût de montage. Autrement dit, la première règle d'or est de ne jamais perdre d'argent sur une représentation, contrairement au spectacle subventionné qui, le plus souvent, ne couvre pas par la billetterie les seuls coûts de plateau.

Rappelons que l'économie de l'exploitation dans une salle doit d'abord tenir compte de deux contraintes que sont le coût du théâtre en ordre de marche (coût annuel recouvrant l'ensemble des charges fixes : personnel permanent, entretien et maintenance, fluides) et la jauge de la salle. Ensuite, il s'agit de consentir une série de coûts dont l'ampleur varie avec les caractéristiques du spectacle et ses modes de production et de valorisation. Le premier est le coût du montage de la production: maîtres d'œuvre, personnel artistique, équipe administrative et technique de production, décors, costumes, accessoires et frais de communication pour le lancement du spectacle. Il s'agit de l'investissement, encore appelé coût de développement. S'ajoutent à celui-ci, les coûts de plateau (artistes en représentation, personnel technique supplémentaire) et enfin les coûts d'ouverture au public : billetterie, personnel d'accueil et de salle, catering, programmes, produits dérivés. La variable stratégique est la recette nette à la représentation (c'est-à-dire droits d'auteurs, royalties et taxes déduits) qui dépend de la jauge de la salle mais aussi de la grille tarifaire, du coefficient de remplissage et de la recette complémentaire par spectateur (bar, achats de programme, de produits dérivés...). Les deux grandes inconnues demeurent la fréquentation et le nombre de représentations, dont dépendent les recettes à la soirée et la durée d'exploitation, donc la période nécessaire aux retours sur investissement et audelà de laquelle le spectacle dégagera du profit. Ce calcul économique n'est pas différent de celui de n'importe quelle entreprise qui lance sur le marché un nouveau produit et doit, tout en maîtrisant ses coûts, réaliser un pari sur le volume des ventes pour un prix espéré en adéqua- tion avec les consentements à payer des consommateurs en se livrant à un banal calcul de point mort.

Le modèle du Musical anglo-saxon, très coûteux, doit donc par nécessité s'inscrire dans la durée la plus longue possible : si elle ne tient pas l'affiche sur une très longue période avec des taux de remplissage très élevés, la production est condamnée au déficit. Par conséquent, la fin d'une production n'est pas programmée à l'avance, selon le principe de l'Open End: on joue tant qu'il y a du public. Si le Roi Lion a ainsi tenu à Paris l'affiche trois saisons, Mama Mia! et Sister Act n'y seront restés que deux saisons et Zorro, une seule. Le taux de remplissage et le nombre de représentations déterminant les règles et les durées de l'amortissement et le moment à partir duquel la production génère du profit, le modèle anglosaxon impose une exploitation sur l'année à raison d'au moins 8 représentations par semaine (soit, à Broadway, un jour de relâche, une représentation en soirée chaque jour et deux matinées mais, à Londres, le nombre de représentations hebdomadaires peut aller jusqu'à 10 , soit 6 soirées et 4 matinées par semaine) et cela, 52 semaines par an, dans des théâtres disposant d'une jauge importante - 1240 places en moyenne pour les 40 théâtres de la Ligue de Broadway. Seuls, 8 théâtres affichent une jauge inférieure à 1000 places et 10 une jauge entre 1500 et 2000 places. À Paris, Stage Entertainment propose à Mogador 7 représentations par semaine.

Si le public attendu n'est plus au rendez-vous, il faut alors aller chercher de nouveaux publics là où ils se trouvent et exploiter le spectacle en tournée : les recettes générées par les tournées de Broadway aux États-Unis sont quasi équivalentes à celles enregistrées à New York, soit près d'un milliard de dollars. Les spectacles proposés en tournée peuvent être des productions spécifiquement conçues pour la tournée ou une production qui a été présentée dans un théâtre et qui connaît ainsi une nouvelle durée d'exploitation. Encore faut-il qu'elle ait été conçue préalablement pour lui permettre de tourner et que les lieux qui l'accueillent le fassent dans des conditions techniques et économiques acceptables.

Enfin, pour les plus grands succès, le spectacle peut être décliné par d'autres producteurs qui achètent une licence dite de première ou de deuxième classe (First ou Second Class Shows). Ces deux types de licence correspondent à des niveaux de contraintes différents. Une licence de deuxième classe donne le droit d'exploiter un titre en en réalisant une nouvelle production, tandis qu'une licence de première classe s'accompagne d'un cahier des charges méticuleux qui impose la reproduction fidèle de la production originale : les auteurs et les tous les ayants droit conservent la maîtrise artistique de l'exploitation.

Pour le Roi Lion, Disney Theatrical se réservant l'exploitation sur le Royaume-Uni et les États-Unis, Stage Entertainment a ainsi acquis une licence First Class Show pour le reste du monde. Le spectacle présenté à Paris était donc la fidèle reproduction de celui de Broadway ou de Londres. L'équipe de production originale (auteur, compositeur, metteur en scène, chorégraphe, éclairagiste, sound designer) a directement participé à l'adaptation et à la mise en place de l'équipe parisienne tandis que 


\section{Des succès colossaux : quelques données...}

Avec un budget de 14 millions de dollars, Wicked (créé le 30 octobre 2003 au Gershwin Theatre à Broadway), à raison de 8 représentations par semaine pendant toute l'année, a généré pour sa première année d'exploitation 56 millions de recettes soit plus d'un million de dollars chaque semaine. À la fin de la première semaine de janvier 2006, Wicked battait le record de la meilleure recette hebdomadaire de l'histoire de Broadway, avec 1611000 dollars, record battu le 26 novembre 2006, avec 1715200 dollars. Bien qu'il s'agisse ici de records, cette logique du retour sur investissement en une année est courante et l'on pourrait multiplier les exemples. Ainsi la production de The Color Purple, créée à Broadway le $1^{\text {er }}$ décembre 2005 et exploitée jusqu'au 24 février 2008, totalisait 910 représentations. Les 11 millions de dollars d'investissement ont été amortis en moins d'une année et le spectacle a généré une recette totale de 103 millions.

Plus la production est lourde, plus le temps nécessaire pour dégager la rentabilité est long mais excède rarement 18 mois. Le spectacle Spiderman, Turn Off the Dark fait figure d'exception avec son budget d'investissement colossal de 65 millions de dollars et des frais d'exploitation hebdomadaires qui seraient de l'ordre d'un million. Pour cette production hors du commun, donnée dans le Foxwoods Theater (1930 places), un rapide calcul supposant un coefficient de remplissage de $96 \%$ et ne prenant en compte que les seules recettes de billetterie conduit à n'espérer le seuil de rentabilité qu'à l'issue de quatre années d'exploitation, bien que le spectacle ait réalisé une recette de 1,878 million la dernière semaine de mars 2013, avec un taux de remplissage de $99 \%$ et un prix moyen par spectateur de 122 dollars. Le producteur, Live Nation ${ }^{1}$, a misé sur d'importantes ventes de produits dérivés : le site web officiel du spectacle donnait ainsi accès à la boutique plusieurs mois avant la première.

1. Leader international sur le marché du spectacle, Live Nation qui, à sa création en 2005, était simple organisateur de concerts, s'est transformé par des rachats successifs en société intégrée contrôlant toute la chaîne du spectacle avec la gestion de lieux (auditoriums, théâtres, arènes, clubs), la vente de billets, la gestion des fans clubs, des produits dérivés et des contrats publicitaires d'artistes. La société travaille ainsi avec Barbra Streisand, le groupe U2, les Rolling Stones, Madonna... Cotée à la Bourse de New York, Live Nation enregistre un chiffre d'affaires de l'ordre de 5 milliards de dollars et emploie quelque 6500 personnes à temps plein. La société organise, avec le concours de quelque 2000 artistes dans 42 pays, 100000 spectacles dont 22000 concerts par an et rassemble 200 millions de spectateurs (source : rapport d'activité 2010 de Live Nation, www.livenation.com/investors).

décors, costumes et mise en scène étaient réalisés à l'identique. La filiale française de Stage ne disposait que d'une marge de manœuvre résiduelle et devait soumettre pour approbation ses idées et projets à Disney Theatrical, qui a gardé un contrôle total, par exemple, des visuels de la communication et des produits dérivés, parfaitement mondialisés. En effet, l'économie de ces spectacles repose aussi sur les produits dérivés. CD, DVD et, dans les meilleurs cas, adaptation cinématographique (Mama Mia! par exemple) sont évidemment des sources de revenus qui peuvent être importantes mais les produits dérivés traditionnels (tee-shirts, photos, livres souvenir, etc.) constituent aussi une source de revenus conséquents. Ainsi, Wicked, spectacle musical s'inspirant de l'univers du célèbre Magicien d'Oz, aurait engrangé quelque 125 millions de dollars grâce aux produits dérivés. Quant au Roi Lion, produits dérivés et licences dans le monde auraient rapporté la somme de 4,2 milliards de dollars.

L'économie complexe de tels spectacles repose aussi sur un examen permanent des coûts et leur maîtrise. C'est ainsi que la troisième saison du Roi Lion à Paris a vu la présentation d'une version allégée pour dégager de la marge et améliorer le retour sur investissement sans pour autant rompre le contrat de confiance avec le spectateur en lui présentant un spectacle au rabais: l'orchestre fut certes réduit mais les orchestrations furent révisées sous l'autorité artistique de l'arrangeur original et le plateau était composé du même nombre d'artistes, les principales économies ayant porté sur la réduction du nombre de doublures. Au-delà, le remplissage de salles à la jauge importante nécessite la maîtrise des outils du marketing, une politique tarifaire sophistiquée particulièrement réactive et un contrôle de la chaîne de valeur depuis l'acquisition du billet jusqu'à l'après spectacle. Stage Entertainment s'est ainsi assuré la vente en ligne de billets en acquérant TopTicketLine.

\section{UN WEST END OU UN BROADWAY À PARIS : Vers UN QUARTIER DU SPECTACLE MUSICAL ?}

Comédie musicale à la française, théâtre musical et spectacles musicaux n'entrent pas en concurrence avec l'opéra, le théâtre traditionnel, la danse contemporaine ou la danse classique et ont leur esthétique propre et leurs spectateurs.

Si la comédie musicale a su prendre sa place dans le paysage du spectacle vivant français, elle reste cependant en deçà de celle qu'elle occupe à l'étranger. À Hambourg, dans un théâtre plus grand que Mogador, le Roi Lion est à l' affiche depuis onze ans et se joue toujours à guichets fermés, tandis qu'à Paris, le Roi Lion a tenu l'affiche trois ans, ce qui est peu au regard de son succès international, mais exceptionnel pour la France.

À New York et à Londres, la notion de district du Musical n'est pas une construction intellectuelle mais bel et bien une réalité, un élément de la dynamique du secteur. Il suffit de regarder le plan du West End pour constater l'extraordinaire proximité des théâtres dans un périmètre qui équivaut peu ou prou à deux arrondissements parisiens. Broadway, West End et des capitales européennes remplissent des salles chaque soir de l'année. Cette forme de spectacle, en développant une logique quasi industrielle, échappe à la fatalité des coûts trop facilement imputée au spectacle vivant. Nous sommes cependant encore loin d'un quartier du spectacle musical à Paris. 


\section{SOURCES}

- Éléments statistiques sur la diffusion des spectacles de variétés et de musiques actuelles, publication annuelle, CNV, Paris. http://www.cnv.fr

- Broadway's Economic Contribution to NYC: 2010-2011 Season, The Broadway League, New York?

- The Demographics of the Broadway Audience 2011-2012, The Broadway League, New York.

- The Economic Impact of Touring Broadway 2008-2009 Season, The Broadway League, New York.

- The Wyndham Report (1998), The Economic Impact of London's West End Theatre, T Travers, The Greater London Group, London School of Economics \& MORI.

- Dominic SHELLARD (2004), Economic Impact Study of UK Theater, University of Sheffield - Arts Council of England, London.
- Success and Foreboding, The Report of the Society of London Theatre 2011.

- GfK-Studie zum Konsumverhalten der Konzert- und Veranstaltungs besucher in Deutschland, IDKV-Bundesverband der Veranstaltungs wirtschaft e.V., 2004.

- Baromètre des investissements publicitaires du secteur des éditions musicales en radio et télévision, année 2009, Observatoire de la musique.

- Cyril Evrard-Noize, Le théâtre musical en France : embellie ou enracinement, Médiamétrie, 2007.

- Catherine RAMPELL, "Spider-Man Economics: Recouping That Initial Investment", New York Times, 14 décembre 2010.

- Catherine RAmPELl, "Spider-Man Economics, Part II", New York Times, 16 décembre 2010

\section{À lire aussi :}

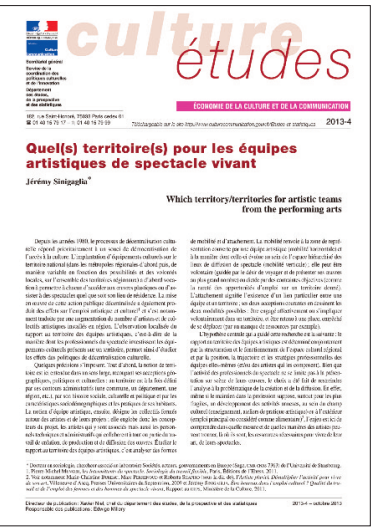

\section{Musiques actuelles : ça part en live}

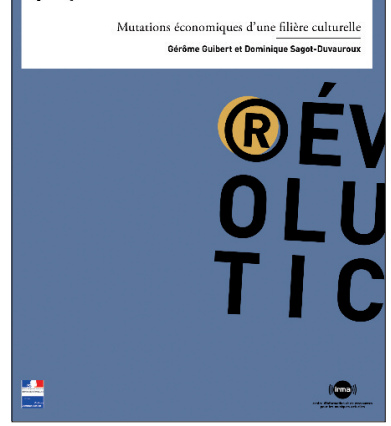

CE-2013-4

\section{Quel(s) territoire(s) pour les équipes artistiques de spectacle vivant}

\section{Jérémy SINIGAGLIA}

Octobre 2013, 12 p.

Comment les équipes artistiques de spectacle vivant déploient-elles leurs activités de création et de diffusion sur un territoire d'implantation ? Par quelles stratégies consolident-elles l'ancrage territorial ou favorisent-elles la mobilité, au sein et au-delà du territoire régional ?

À partir de l'exploitation de données administratives et d'une enquête qualitative menée en Alsace et en Lorraine, l'étude distingue deux réseaux de diffusion qui structurent l'espace culturel régional : un réseau primaire qui regroupe les grandes salles privées et les salles labellisées par l'État, et un réseau secondaire où l'on retrouve des établissements soutenus par l'État et les collectivités territoriales et des salles privées de moindre envergure commerciale. En outre, un réseau qualifié de parallèle offre également des opportunités de diffusion aux artistes, à des conditions d'emploi et de rémunération satisfaisants : réseau scolaire et employeurs occasionnels du secteur privé, pour de l'animation musicale par exemple.

Résidant majoritairement dans les métropoles régionales qui concentrent aussi équipements et employeurs culturels, les équipes artistiques de spectacle vivant déploient des logiques différenciées qui renforcent l'attachement ou la mobilité, subis ou choisis selon les cas. Alors que l'ancrage territorial des équipes artistiques est nécessaire pour solliciter l'obtention d'un financement auprès des collectivités territoriales et peut aussi être le signe d'un attachement positif, la mobilité au-delà du territoire d'implantation permet d'accéder au réseau primaire de diffusion, de diversifier les employeurs et d'élargir la notoriété.

\section{Musiques actuelles: ça part en live}

\section{Gérôme Guibert et Dominique Sagot-Duvauroux}

Dans un contexte de crise qui déstabilise toute la filière musicale, cet ouvrage propose une analyse précise de l'économie des concerts de musiques actuelles. Du producteur à la salle de spectacles ou au festival, il décrit l'organisation de la chaîne d'acteurs qui contribuent à la réalisation d'un spectacle et les relations contractuelles qui les lient, variables selon le degré de notoriété des artistes.

De tailles et de statuts très divers, les structures de spectacle vivant investies dans les musiques actuelles forment un ensemble hétérogène et fragile dont l'organisation diffère sensiblement de celle des pays anglo-saxons. La France se singularise ainsi par le rôle du producteur « employeur du plateau artistique » et par l'indépendance, le nombre et la diversité des entreprises, petites et moyennes, qui produisent des concerts pour des artistes confirmés ou en développement.

Les auteurs soulignent les interdépendances croissantes entre les acteurs de l'industrie phonographique et de l'organisation de concerts, qui se traduisent par d'importants mouvements de concentration. De nouveaux acteurs - majors du disque, multinationales de l'entertainment ou de la communication - occupent désormais des positions stratégiques.

L'ouvrage met également en avant les complémentarités entre circuits commerciaux, associatifs et publics, qui contribuent, chacun à des stades différents de la carrière des artistes, à la valorisation de la musique. 


\section{RÉSUMÉ}

Née en France à la fin du XIX ${ }^{\mathrm{e}}$ siècle avec l'opérette, la comédie musicale a fait le bonheur du public anglo-saxon, au point d'incarner l'identité de certains quartiers de Londres (West End) et de New York (Broadway), tandis que le genre subissait un certain désamour auprès du public français. Les succès de Starmania en 1979 puis de Notre-Dame de Paris en 1998 ont relancé l'intérêt des créateurs, des programmateurs et du public français pour le spectacle musical qui, à la fin des années 2000, tient de nouveau le haut de l'affiche et remplit les salles.

Trois types de spectacles musicaux peuvent être caractérisés, qui répondent à des exigences artistiques différentes et auxquels correspondent des logiques financières et des modèles économiques bien distincts : la comédie musicale à la française, proche du spectacle de variétés, le théâtre musical dont le modèle économique est similaire à celui du spectacle vivant subventionné, et la comédie musicale à l'anglo-saxonne, représentée en France par le théâtre Mogador, où prédomine une logique industrielle du spectacle.

Le genre est désormais investi par certains théâtres qui en ont fait leur marque et le spectacle musical connaît de beaux succès populaires. Pour autant, l'activité française reste modeste au niveau national (moins de $10 \%$ de la fréquentation totale des représentations payantes) et en comparaison de l'activité aux États-Unis, au Royaume-Uni ou même en Allemagne.

\section{ABSTRACT}

From its inception in France at the end of the 20th century with the operetta, musical theatre came to captivate AngloSaxon audiences, to the extent that it has shaped the identity of certain areas of London (the West End) and New York (Broadway); meanwhile, the genre fell from favour with French audiences. The success of Starmania in 1979 and NotreDame-de-Paris in 1998 reignited the interest of creators, promoters and French audiences in musical theatre and towards the end of the 2000s, it began to top the ratings and play to packed houses.

There are three main types of musical theatre, corresponding to different artistic demands and each with their own discrete financial schemes and economic models: the French comédie musicale, which is similar to the variety show; musical theatre, whose economic model is similar to that for subsidised performing arts, and "Anglo-Saxon" style musical theatre, represented in France by the Mogador theatre where an industrial approach to live entertainment prevails.

However the genre has been promoted by certain theatres which have made their mark upon it and musicals have seen some spectacular popular success stories. Nevertheless, such activity in France remains fairly low at national level (less than 10\% of total audiences for paying productions) and in comparison with such activity in the US, United Kingdom and even Germany.

\section{Tous les documents publiés par le DEPS sont téléchargeables sur http://www.culturecommunication.gouv.fr/Etudes-et-statistiques et sur www.cairn.info}

Pour recevoir régulièrement les publications du DEPS et pour toute demande d'information :

contact.deps@culture.gouv.fr 